\title{
STANDARD BREAST-CONSERVING VERSUS ONCOPLASTIC SURGERY: A LOCOREGIONAL RECURRENCE COMPARISON
}

\author{
Juliane Dal Vesco ${ }^{1}$, Mario Casales Schorr ${ }^{1}$ \\ 'Nossa Senhora da Conceição Hospital - Porto Alegre (RS), Brazil.
}

Objectives: Oncoplastic surgery has provided new options for breast conserving surgery - including cases of locally advanced diseases and multifocal tumors - with better aesthetic results, thus contributing to a better quality of life for patients. Oncological safety is still being discussed, mainly with reference to the incidence of local recurrences as well as the influence of breast remodeling on the accuracy of imaging tests and the planning of adjuvant radiotherapy. Methods: This is a retrospective, cross-sectional study of patients who underwent breast conservation surgery for invasive and noninvasive breast cancer at the Nossa Senhora da Conceição Hospital (HNSC) from 2010 to 2017. Results: A total of 956 cases of breast conservation surgery were considered for our study: 90 cases of oncoplastic surgery and 866 cases of standard conservation surgery. The mean follow-up time was 51 months. Standard breast conservation surgery was related to postmenopausal patients, aged 70 years or older. Significantly, oncoplastic surgery was more frequently indicated for patients between 31 and 39 years old. Tumor size, multicentricity, and axillary involvement were identified in greater numbers in oncoplastic surgery. There was no statistical difference between local recurrence and death in relation to breast cancer. Conclusions: Oncoplastic surgery is an individualized surgical treatment which aims to maintain oncological safety while achieving better aesthetic results and minimizing the mutilation of female patients affected by breast cancer. 